\title{
Ammonium tetrathiomolybdate enhances the antitumor effects of cetuximab via the suppression of osteoclastogenesis in head and neck squamous carcinoma
}

\author{
AYAKA MORISAWA $^{1}$, TATSUO OKUI ${ }^{1}$, TSUYOSHI SHIMO ${ }^{1}$, SOICHIRO IBARAGI $^{1}$, YUKA OKUSHA $^{2}$, \\ MITSUAKI ONO $^{3}$, THI THU HA NGUYEN ${ }^{4}$, NUR MOHAMMAD MONSUR HASSAN ${ }^{5}$ and AKIRA SASAKI ${ }^{1}$ \\ Departments of ${ }^{1}$ Oral and Maxillofacial Surgery, ${ }^{2}$ Dental Pharmacology, ${ }^{3}$ Molecular Biology and Biochemistry, and \\ ${ }^{4}$ Oral Rehabilitation Regenerative Medicine, Okayama University Graduate School of Medicine, Dentistry, \\ and Pharmaceutical Sciences, Okayama 700-8525, Japan; ${ }^{5}$ School of Dentistry and Health Sciences, \\ Charles Sturt University, Sydney, Orange NSW, Australia
}

Received September 21, 2017; Accepted January 4, 2018

DOI: $10.3892 /$ ijo.2018.4242

\begin{abstract}
Head and neck squamous cell carcinoma (HNSCC) poses a significant challenge clinically where one of the mechanisms responsible for the invasion into facial bones occurs via the activation of osteoclasts. Copper has been demonstrated to play a key role in skeletal remodeling. However, the role of copper in cancer-associated bone destruction is thus far unknown. Lysyl oxidase (LOX) is a copper-dependent enzyme that promotes osteoclastogenesis. In the present study, we investigated the effects of copper on HNSCC with bone invasion by the copper chelator, ammonium tetrathiomolybdate (TM) in vitro and in vivo. We demonstrate that TM blocks the proliferation of HNSCC cells, inhibits LOX activation and decreases the expression of the receptor activator of nuclear factor- $\kappa \mathrm{B}$ ligand (RANKL) in osteoblasts and osteocytes, subsequently suppressing bone destruction. These findings suggest that copper is a potential target for the treatment of HNSCCs associated with bone destruction.
\end{abstract}

\section{Introduction}

Head and neck squamous cell carcinoma (HNSCC) frequently invades the facial bones, and this invasion is a prognostic factor for poor clinical outcomes $(1,2)$. Bone resection is a treatment that often leads to the post-operative disruption of speech and swallowing functions, and thus poses a significant challenge

Correspondence to: Dr Tatsuo Okui, Department of Oral and Maxillofacial Surgery, Okayama University Graduate School of Medicine, Dentistry, and Pharmaceutical Sciences, 2-5-1 Shikata-cho, Kita-ku, Okayama 700-8525, Japan

E-mail: tatsuookui0921@gmail.com

Key words: copper, head and neck squamous cell carcinoma, bone invasion, osteoclastogenesis to the quality of life of patients with HNSCC presenting with facial bone invasion. Cancer cells have been demonstrated to secrete significant amounts of growth factors, which promotes osteoclastogenesis (3). Therefore, it is critical that novel approaches should be evaluated for the treatment of bone destruction in advanced HNSCC. Copper is known as a key factor for cellular metabolism, neuronal transmission and bone remodeling (4,5). Copper metabolic disorder induces Wilson's disease, a rare inherited disorder that causes copper to accumulate in the liver, brain and other vital organs (6). The copper chelator, ammonium tetrathiomolybdate (TM), is used for the treatment of copper metabolic disorder and Wilson's disease. In addition, recent research has revealed that copper chelators exert an antitumor effect against several cancer types, such as breast cancer with lung metastases and head and neck cancer $(7,8)$.

Copper is a factor that binds to selected enzymes and functions to increase their activation. For example, Lysyl oxidase (LOX) is the prototypical member of copperdependent enzymes whose documented function is to oxidize primary amine substrates to reactive aldehydes (9). The most well-characterized role of LOX is in the remodeling of the extracellular matrix (ECM) through the oxidative deamination of peptidyl lysine residues in collagens and elastin to facilitate covalent cross-linking (10). It has also been reported that LOX is essential for bone remodeling via the regulation of receptor activator of nuclear factor- $\kappa \mathrm{B}$ ligand (RANKL) expression on bone marrow stromal cells $(11,12)$. Cancer cells release significant amounts of LOX (13). This copper-dependent LOX activation may promote bone resorption; however, the role of copper in bone resorption in HNSCC remains unclear and thus requires clarification.

In the present study, we thus aimed to determine the role of copper in bone resorption in HNSCC. To the best of our knowledge, we are the first to provide evidence that the copper chelator, TM, exerts an anti-bone destruction effect against cancer-induced bone resorption and that TM enhances the antitumor effect of a clinically validated anticancer agent on HNSCC associated with bone invasion. 


\section{Materials and methods}

Cell lines and culture conditions. The human HNSCC cell lines, HSC-2 (\#JCRB0622), HSC-3 (\#JCRB0523) and SAS (\#JCRB0260), were obtained from the Human Science Research Resources Bank (Osaka, Japan). All cell lines were cultured in Dulbecco's modified Eagle's medium (DMEM) supplemented with $10 \%$ heat-inactivated fetal bovine serum (FBS). Primary fibroblasts were obtained from Cosmo Bio (\#SCR2620, Tokyo, Japan).

Primary osteoblasts, osteocytes, bone marrow cells (obtained as described below), and fibroblasts were cultured in alpha-modification of minimum essential medium ( $\alpha$-MEM). T cells (obtained as described below) were cultured in RPMI-1640 medium supplemented with $10 \%$ FBS, $1 \%$ penicillin-streptomycin, 10 mM 2-mercaptoethanol (Sigma, St. Louis, MO, USA) and Pyruvic acid (Wako, Osaka Japan). All of the above-cited cell lines were characterized by genotyping at cell banks. All cell lines were cultured in an atmosphere of $10 \% \mathrm{CO}_{2}$ at $37^{\circ} \mathrm{C}$.

Osteoclastogenesis assay. Bone marrow cells were obtained from the femurs and tibiae of 4-week-old male C57BL/6 mice $(\mathrm{n}=2)$, purchased from Charles River Laboratories (Yokoyama, Japan). Under anesthesia with $0.4 \mathrm{mg} / \mathrm{kg}$ of medetomidine, $4.0 \mathrm{mg} / \mathrm{kg}$ of midazolam and $5.0 \mathrm{mg} / \mathrm{kg}$ of butorphanol, the mice were sacrificed by cervical dislocation. After the leg was cut, muscle and connective tissue was removed from the tibiae and femurs. Both ends of the femur and tibiae were then clipped with a scissors. A 5 cc syringe was filled using a 27 gauge needle with PBS, and a needle was inserted into one end and the bone marrow was flushed out the other end. The flushed bone marrow cells wre corrected in a $50 \mathrm{cc}$ tube. The bone marrow cells were washed twice by centrifugation $(125 \mathrm{x} \mathrm{g}$, $10 \mathrm{~min})$ in $4^{\circ} \mathrm{C} \mathrm{PBS}$. The cells were the nincubated in $\alpha$-MEM in $10 \mathrm{~cm}$ culture dishes in the presence of macrophage colonystimulating factor (M-CSF) $(10 \mathrm{ng} / \mathrm{ml})$ for $24 \mathrm{~h}$. Non-adherent cells were transferred to 24 -well plates $\left(2 \times 10^{6}\right.$ cells/well). The cells were treated with vitamin $\mathrm{D}_{3}, 1,25$-Dihydroxy $\left(10^{-8} \mathrm{M}\right)$ and $\operatorname{TM}(0.1,1,2.5$ and $5 \mu \mathrm{M})$ for 9 days.

Purification of osteoclast progenitors. Bone marrow cells were washed twice by centrifugation $(125 \mathrm{x} \mathrm{g}, 10 \mathrm{~min})$ in $20 \mathrm{ml}$ of $4^{\circ} \mathrm{C}$ sterile phosphate-buffered saline (PBS) supplemented with $0.5 \%$ bovine serum albumin. The cell pellet was resuspended, and the cells were magnetically labeled by the addition of anti-CD11b microbeads (Miltenyi Biotec $\mathrm{GmbH}$, Bergisch Gladbach, Germany). The cells were then incubated for $30 \mathrm{~min}$ on ice and then washed by centrifugation $(125 \mathrm{x} \mathrm{g}$, $10 \mathrm{~min}$ ) with a volume of $4^{\circ} \mathrm{C}$ buffer that was 10 -fold that of the labeling volume and resuspended. $\mathrm{CD} 11 \mathrm{~b}^{+}$cells were depleted using an MD depletion column (Miltenyi Biotec $\mathrm{GmbH}$ ). A total of $1 \times 10^{5}$ murine $\mathrm{CD} 11 \mathrm{~b}^{+}$bone marrow cells/well were plated in a 24-well plate. The cells were treated with RANKL (50 ng/ml) and M-CSF (30 ng/ml) and the desired amount of $\mathrm{TM}(0.1,1,2.5$ and $5 \mu \mathrm{M})$ for 9 days. Following 9 days of incubation, the cells were fixed and stained for tartrateresistant acid phosphatase activity using the acid phosphatase, leukocyte (TRAP) kit (\#A386A, Merck KGaA, Darmstadt, Germany), and the number of TRAP-positive multinucleate cells (i.e., a nuclear number $>3$ ) in each well was then counted.
Cell proliferation assay. The HSC-2, HSC-3 and SAS cells were each plated in 6-well plates at a density of $5 \times 10^{3}$ cells per well and treated with the TM $(1,5 \mu \mathrm{M})$ or with an equivalent volume of the diluent (DMSO) as a control for 5 days. Osteoblasts, osteocytes and fibroblasts were each plated in 6 -well plates at a density of $1 \times 10^{4}$ cells per well and treated with $\mathrm{TM}(1,5 \mu \mathrm{M})$ for $48 \mathrm{~h}$. $\mathrm{CD}^{+} \mathrm{T}$ cells were isolated by the magnetic sorting system. The cells from the homozenized spleens of two C57BL/6J mice were incubated with CD4 antibody-conjugated microbeads (eBioscience, San Diego, CA, USA) and sorted with MD depletion column (Miltenyi Biotec). The cells were stimulated with anti-CD3 monoclonal antibody (Cat. no. 16-0031, $5 \mu \mathrm{g} / \mathrm{ml}$ ), CD28 antibody (Cat. no. 16-0281, $2 \mu \mathrm{g} / \mathrm{ml}$ ) (Affymetrix, Santa Clara, CA, USA) and TM $(1,5 \mu \mathrm{M})$ for $48 \mathrm{~h}$. The cell number was then counted with trypan blue assay. Osteoblasts were obtained following the method of Teramachi et al (14). After flushing the bone marrow from the tibiae of $3 \mathrm{C} 57 \mathrm{BL} / 6 \mathrm{~J}$ mice, the tibiae were cultured in $\alpha \mathrm{MEM}$ for 7-10 days in $60-\mathrm{mm}$ dishes until the cells growing out of the bones formed a confluent monolayer. The original bone was removed, and the cells grown out of the the bone were treated with $0.25 \%$ trypsin and $0.05 \%$ EDTA for $10 \mathrm{~min}$ at $37^{\circ} \mathrm{C}$. These cells were used as primary osteoblas without further passage. Osteocytes were obtained following the method of Shah et al (15). After flushing the bone marrow from the tibiae of the $2 \mathrm{C} 57 \mathrm{BL} / 6 \mathrm{~J}$ mice, the bones were dissected into 1-2-mm sized sections. The bone sections were then incubated for $25 \mathrm{~min}$ in collagenase (300 units/ml) and $0.25 \%$ EDTA $(5 \mathrm{mM})$ and the collagenase was removed and discarded. These processes were repeated 8 times. The cells from the bone sections were cultured as osteocytes without further passage. These cells were classified as osteocytes by measuring dentin matrix acidic phosphoprotein 1 (DMP-1) expression by western blot analysis (data not shown).

Copper concentration measurement assay. The copper concentrations in the culture medium and serum, collected from the tail vein of the mice inoculated with the cancer cells at the time of sacrifice were measured using the Metallo Assay Copper Assay kit (Funakoshi, Tokyo, Japan). The samples, buffer and chelate color solution were mixed and incubated for $10 \mathrm{~min}$ at room temperature. Subsequently, the absorbance was read at a $580 \mathrm{~nm}$ wavelength using a microplate reader (SH-1000, Hitachi, Tokyo, Japan).

LOX activity assay. The HSC-2 and SAS cells were cultured in DMEM with increased copper ion $(10 \mu \mathrm{M})$ in the presence or absence of TM for $24 \mathrm{~h}$. Conditioned medium and serum collected from the mice inoculated with the cancer cells were tested using a LOX activity kit (Cat. no. ab112139, Abcam, Cambridge, MA, USA). The samples and LOX reaction mix solution were mixed and incubated for $30 \mathrm{~min}$ at room temperature. Subsequently, the fluorescence increase was measured on a microplate reader (Gemini EM microplate reader, Molecular Devices, Sunnyvale, CA, USA) at excitation and emission wavelengths of 550 and $600 \mathrm{~nm}$. These conditioned media were used in the following experiment.

Western blot analysis. The osteocytes and osteoblasts were cultured in the above-mentioned conditioned media (30\%) 
A

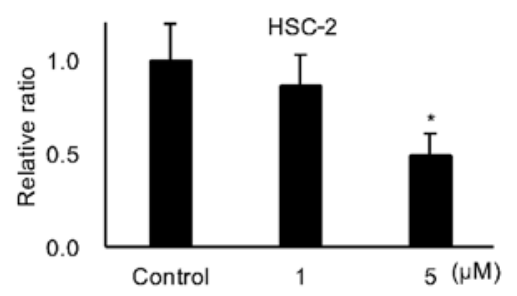

C
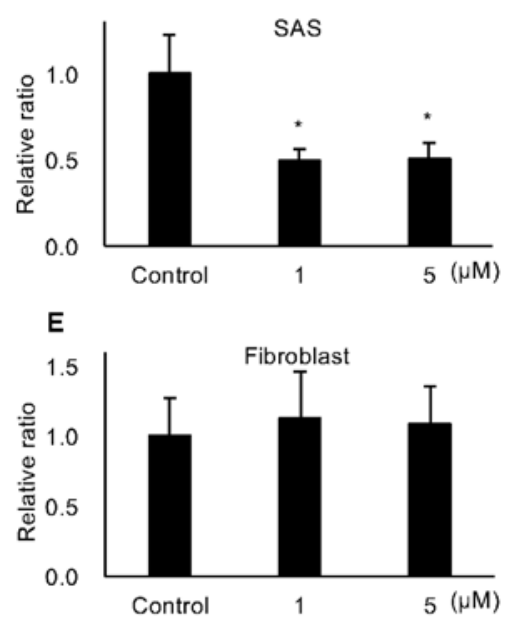

G

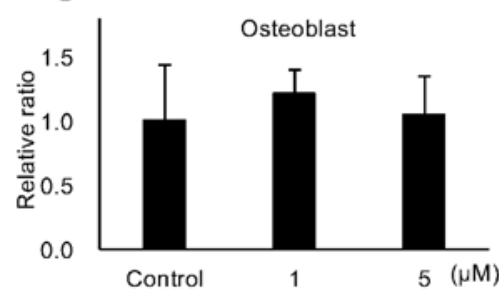

B

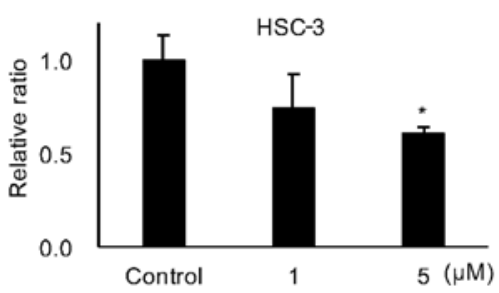

D

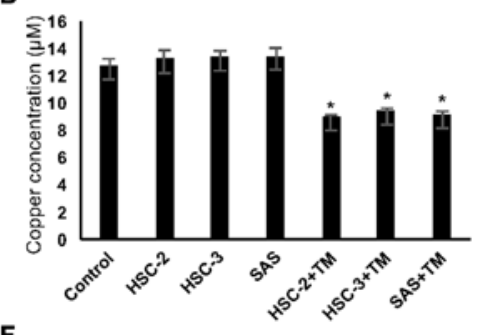

$\mathbf{F}$

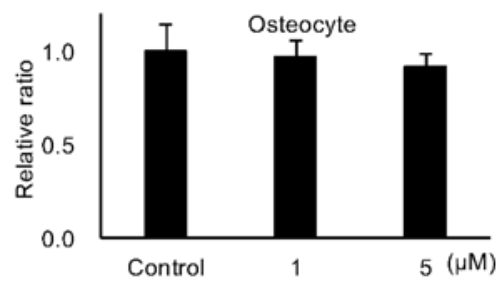

H

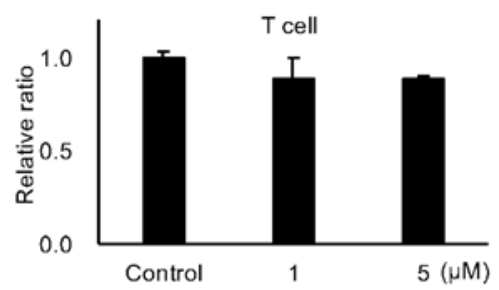

Figure 1. Effects of ammonium tetrathiomolybdate (TM) on the proliferation of HNSCC and bone microenvironment cells in vitro (A) HSC2 cells, (B) HSC3 cells, (C) SAS cells, (E) osteoblasts, (F) osteocytes, (G) fibroblasts, and (H) T cells were plated in triplicate and treated with 0,1 , or $5 \mu \mathrm{M}$ TM for $48 \mathrm{~h}$. Error bars indicate the means $\pm \mathrm{SD}$; ${ }^{*} \mathrm{P}<0.05$ vs. control. (D) Copper concentration in culture medium from HNSCC cells treated with TM $(5 \mu \mathrm{M})$ for $48 \mathrm{~h}$. Error bars indicate the means $\pm \mathrm{SD} ;{ }^{*} \mathrm{P}<0.05$ vs. untreated cells.

for $24 \mathrm{~h}$. The cell culture conditioned media $(25 \mu \mathrm{l})$ were mixed with 4X Laemmli sample buffer (Bio-Rad, Hercules, CA, USA) and boiled at $95^{\circ} \mathrm{C}$ for $5 \mathrm{~min}$. The samples were electrophoresed in 4-12\% SDS-PAGE gels and the proteins were transferred onto membranes (Immobilon-P; Millipore, Bedford, MA, USA). The membranes were incubated with primary and secondary antibodies according to the ECL chemiluminescence protocol (RPN2109; Amersham Biosciences, Buckinghamshire, UK) to detect secondary antibody binding. Antibodies against RANKL (Cat. no. sc-377079, 1:1,000, Santa Cruz Biotechnology (Dallas, TX, USA) was used as a primary antibody and HRP-conjugated anti-mouse antibody (Cat. no. 7076, 1:2,000, Cell Signaling Technology, Danvers, MA, USA) was used as the secondary antibody.

Immunohistochemical analysis. The tibial bone and soft tumor was fixed in $10 \%$ formalin, decalcified and then embedded in paraffin. Serial sections were then prepared (3- $\mu \mathrm{m}$-thick). For immunohistochemical analysis, the specimens were incubated with antibody (CD-31, 1:50, Cat. no. ab28364, Abcam), (Ki67, 1:400, Cat. no. 9129, Cell Signaling Technology), (EGFR, 1:50,
Cat. no. 4267, Cell Signaling Technology), (p-EGFR, 1:200, Cat. no. 3777, Cell Signaling Technology), (RANKL, 1:100, Cat. no. sc-377079, Santa Cruz Biotechnology) overnight at $4^{\circ} \mathrm{C}$, followed by 3 washes with TBS. The slides were then treated with a streptoavidin-biotin complex [EnVision System labelled polymer, horseradish peroxidase (HRP); Dako, Carpinteria, CA, USA] for $60 \mathrm{~min}$ at a dilution of 1:100. The immunoreaction was visualized by using a DAB substratechromogen solution (Dako Cytomation Liquid DAB Substrate Chromogen System; Dako). The cells were counted using a microscope and evaluated.

Animal experiments. Mouse models of bone invasion by human oral squamous cell carcinoma were established in 5-week-old female BALB/c nude mice (each group, $n=5$; total, $n=20$, mean body weight, $19.5 \mathrm{~g}$; Charles River Laboratories) by the inoculation of $1 \times 10^{5} \mathrm{HSC}-2$ cells into the bone marrow space of the left tibial metaphysis. At 7 days after tumor cell inoculation, the mice were divided into 4 groups (control, cetuximab-treated, TM-treated, and TM- and cetuximab-treated). The cetuximab group was treated with an intraperitoneal injection of $100 \mu \mathrm{l}$ of 
a solution containing cetuximab $(1 \mathrm{mg} / \mathrm{kg})$ in PBS or PBS alone twice a week for 5 weeks. The TM group was orally administered a $100 \mu l$ solution containing TM $(1 \mathrm{mg})$ in distilled deionized water (DDW) or DDW alone 5 times a week for 5 weeks. Under anesthesia with $0.4 \mathrm{mg} / \mathrm{kg}$ of medetomidine, $4.0 \mathrm{mg} / \mathrm{kg}$ of midazolam and $5.0 \mathrm{mg} / \mathrm{kg}$ of butorphanol, the hind limb long bones of the nude mice that had been injected with the cancer cells were excised, fixed in $10 \%$ neutralbuffered formalin. Osteolytic bone destruction was assessed on radiographs. The bones were placed against films $(22 \times 27 \mathrm{~cm}$; Fuji industrial film FR: Fuji Photo Film Co. Ltd., Tokyo, Japan), and exposed to soft X-rays at $35 \mathrm{kV}$ for $15 \mathrm{sec}$ by the use of a Sofron apparatus (Sofron, Tokyo, Japan). The radiolucent bone lesions were observed microscopically (IX81, Olympus, Tokyo, Japan), and the areas were quantified with Lumina Vision/OL (Mitani, Tokyo, Japan). A micro-CT image was obtained with SKYSCAN (Bruker Japan, Kanagawa, Japan). Subsequently, the bone was decalcified and embedded in paraffin. Serial sections ( $3 \mu \mathrm{m}$-thick) were cut cross-sectionally, and the sections were stained with TRAP stain.

Human oral squamous cell carcinoma xenografts were established in 5-week-old male BALB/c nude mice (each group, $n=5$; total, $n=20$, Charles River Laboratories) by the inoculation of $1 \times 10^{6} \mathrm{HSC}-2$ cells into the dorsal flank. At 7 days after tumor cell inoculation, the mice were divided into 4 groups (control, cetuximab-treated, TM-treated, and TM- and cetuximab-treated). The mice were treated with an intraperitoneal injection of $100 \mu 1$ of solution containing cetuximab $(1 \mathrm{mg} / \mathrm{kg})$ in PBS or PBS alone twice a week for 5 weeks. The mice were treated with orally adminisitration of $100 \mu \mathrm{l}$ of a solution containing TM $(1 \mathrm{mg})$ or DDW, 5 times a week for 5 weeks. The tumor volume (cubic $\mathrm{mm}$ ) was calculated using the following equation: $4 \pi / 3 \times \mathrm{xr} 1 / 2 \times \mathrm{xr} 2 / 2^{2}$, where $\mathrm{rl}$ is the longitudinal diameter, and $\mathrm{r} 2$ is the transverse diameter. At 5 weeks after tumor cell inoculation, all the mice were then sacrificed, and the volume of tumors was measured.

All the animal experimental protocols were approved by the Ethics Review Committee for Animal Experimentation of the Okayama University Graduate School of Medicine and Dentistry with the following approval numbers: OKU-2016055 (for human oral squamous cell carcinoma xenografts), OKU2016060 (isolation of bone-related cells) and OKU-2016056 (inoculation of cancer cells into bone marrow).

Statistical analysis. The experiments were performed in quadruplicate. Data were analyzed using an unpaired Student's t-test for the analysis of two groups, and one-way ANOVA with Bonferroni and Dunnett's post hoc tests for the analysis of multiple group comparisons using SPSS statistical software. The results are expressed as the means $\pm S D$. A value of $P<0.05$ was considered to indicate a statistically significant difference.

\section{Results}

TM suppresses the growth of oral squamous cell carcinoma cells. To examine the antitumor effects of TM against oral squamous cell carcinoma in vitro, we performed a trypan blue staining assay. As shown in Fig. 1A-C, TM significantly reduced the number of viable HSC-2, HSC-3 and SAS cells proportionately with the increasing concentrations 5 days
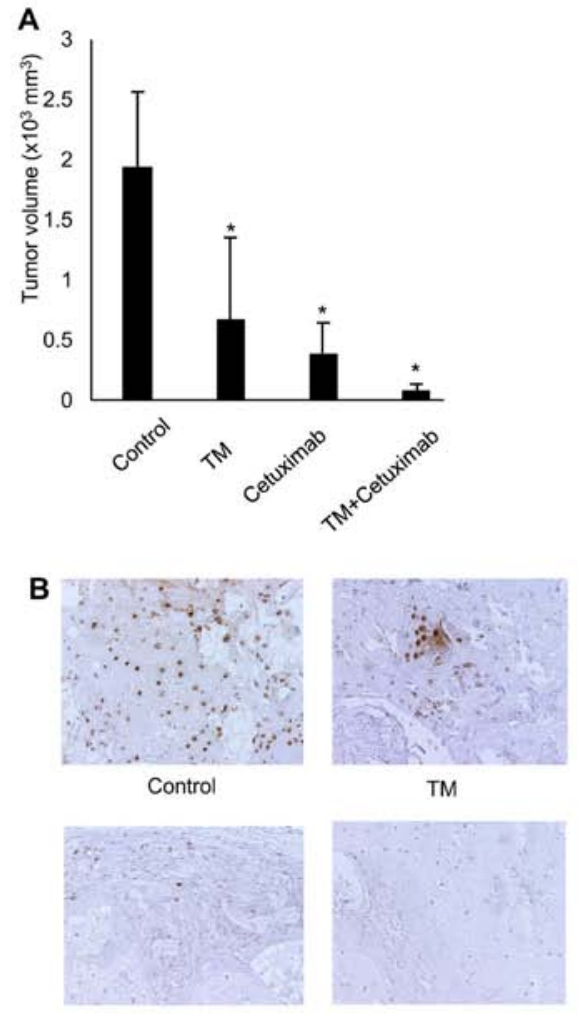

Cetuximab

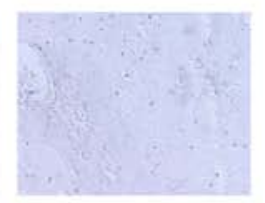

$\mathrm{TM}+$ Cetuximab

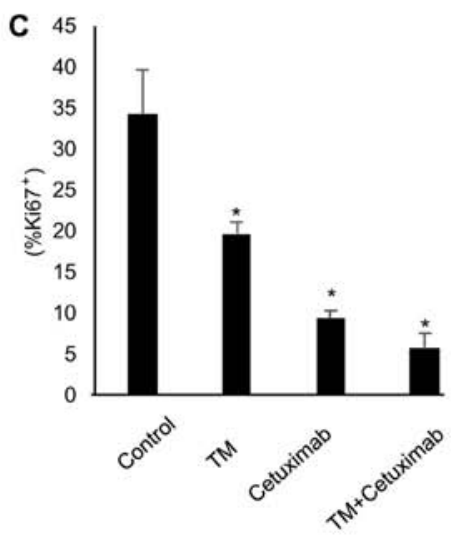

Figure 2. Effects of TM and cetuximab on oral squamous cell carcinoma cells grown in mice. (A) Tumor growth is expressed as the mean tumor volume \pm SD; ${ }^{*} \mathrm{P}<0.05$ vs. control. (B) Immunohistochemical analysis of Ki67 in xenograft tumors. (C) The percentage of Ki67-positive cells in each section; ${ }^{*} \mathrm{P}<0.05$ vs. control.

after treatment. In the same experiment, the concentrations of copper ion in the conditioned media of the HSC-2, HSC-3 and SAS cells treated with $5 \mu \mathrm{M}$ TM for $72 \mathrm{~h}$ was decreased by approximately $30 \%$ (Fig. 1D). By contrast, TM did not affect the proliferation of fibroblasts, osteoblasts, osteocytes and $\mathrm{T}$ cells, which are components of the bone microenvironment (Fig. 1E-H).

To examine the antitumor effects of TM in vivo, we established an HNSCC xenograft tumor derived from HSC-2 cells in nude mice. The mice were treated with TM $(1 \mathrm{mg}$; 5 times a week) and/or cetuximab ( $1 \mathrm{mg} / \mathrm{kg}$; twice a week) for 5 weeks beginning at 7 days after tumor cell inoculation, and the tumor volume was measured at day 35 . As shown in Fig. 2A, the HSC-2 xenograft tumor volumes were significantly 
A

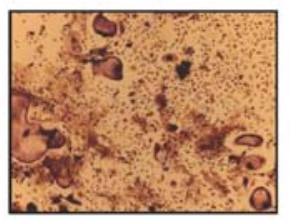

Control

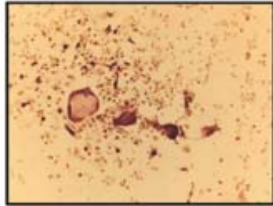

$2.5 \mu \mathrm{M}$

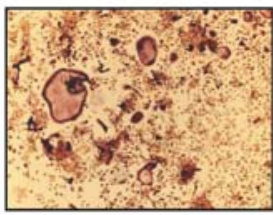

$0.1 \mu \mathrm{M}$

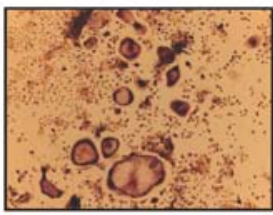

$1 \mu \mathrm{M}$

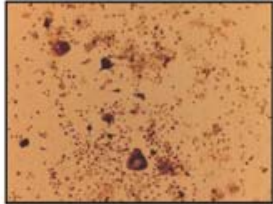

$5 \mu \mathrm{M}$

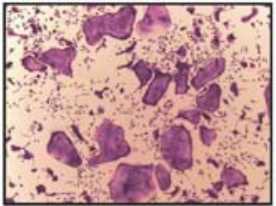

RANKL(100 ng/ml)
B

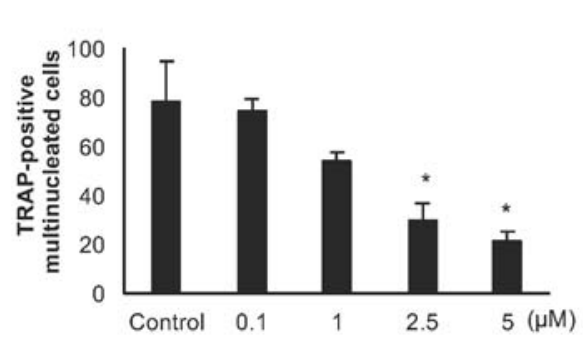

C

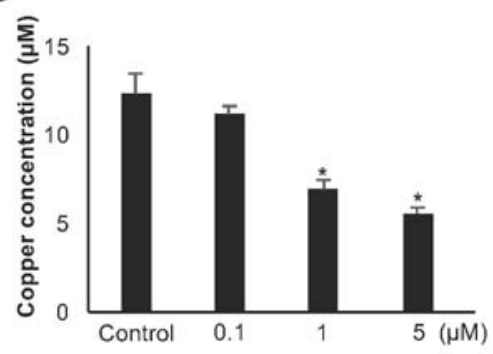

D

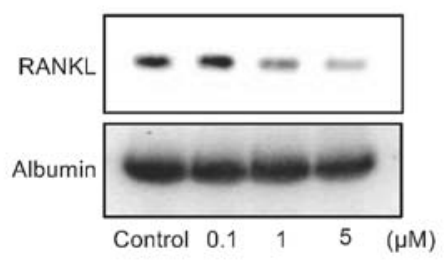

E

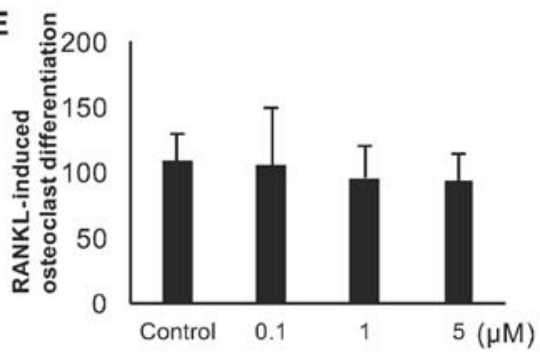

Figure 3. Effect of ammonium tetrathiomolybdate (TM) on osteoclastogenesis. (A and B) Total bone marrow cells were cultured with TM $(0,0.1,1,2.5$, or $5 \mu \mathrm{M}$ ) in the presence of vitamin $\mathrm{D}_{3}, 1,25$-Dihydroxy $\left(10_{-8} \mathrm{M}\right)$ in a 24-well plate for 9 days. (B) The numbers of TRAP-positive multinucleated cells (nuclear number $>3$ ) were counted as osteoclasts ( $\mathrm{y}$-axis). Data are the means $\pm \mathrm{SD} ;{ }^{*} \mathrm{P}<0.05$ between the indicated groups. (C) Copper concentration in bone marrow cell cultured medium in the presence of vitamin $\mathrm{D}_{3}, 1,25$-Dihydroxy $\left(10^{-8} \mathrm{M}\right)$ with or without $\mathrm{TM}(0.1,1,5 \mu \mathrm{M})$ for 3 days. Data are means $\pm \mathrm{SD}$; ${ }^{*} \mathrm{P}<0.05$ vs. control (D) RAKNL expression in bone marrow cell culture medium in the presence of vitamin $\mathrm{D}_{3}, 1,25$-Dihydroxy $\left(10^{-8} \mathrm{M}\right)$ with or without $\mathrm{TM}(0.1,1,5 \mu \mathrm{M})$ for 3 days. (E) CD11b-positive pre-osteoclasts were cultured with TM $(0,0.1,1,5 \mu \mathrm{M})$ in the presence of $30 \mathrm{ng} / \mathrm{ml} \mathrm{M}$-CSF and $50 \mathrm{ng} / \mathrm{ml} \mathrm{RANKL}$ in a $24-w e l l$ plate for 9 days. TRAP-positive multinucleated cells (nuclear number $>3$ ) were counted as osteoclasts. Data are the means \pm SD.

decreased in the TM-or cetuximab-treated mice compared to the untreated mice. No significant toxicity was observed during the treatment period. At sacrifice after the treatment period with TM or cetuximab, the tumors were excised and examined histologically. Immunohistochemical analysis revealed a significant decrease in the number of Ki67-positive tumor cells in the HSC-2 tumor sections from the TM-treated and cetuximab-treated mice (Fig. 2B and C). TM treatment led to a positive trend by enhancing the antitumor effects of cetuximab (cetuximab single treatment vs. combined treatment, $\mathrm{P}=0.057$ ). TM or cetuximab did not cause any body weight loss at the end of experiment [mean body weight $(\mathrm{g})$ : control, 23.01; TM, 23.15; cetuximab, 24.15; and TM + cetuzimab, 22.8]. None of the animals experienced $>20 \%$ decrease in body weight during the experiment. However, no statistically significant differences were observed between single treatment and combination treatment with TM and cetuximab as regards by tumor volume in this soft tissue tumor model. These results suggest that antitumor effects of TM require further evaluation in soft tissue HNSCC models in order to definitively evaluate its direct anticancer activity.

TM suppresses osteoclastformation. Copper ions are known to play a key role in bone remodeling (5); however, the effects of copper chelating on bone cancer remain unknown. To examine the effects of TM on osteoclast formation, we treated murine total bone marrow cells harvested from mouse tibias with vitamin $\mathrm{D}_{3}\left(1 \times 10^{-8} \mathrm{M}\right)$ in the presence or absence of TM for 5 days. TM inhibited the number of TRAP-positive multinucleated osteoclasts in a dose-dependent manner (Fig. 3A and B). 

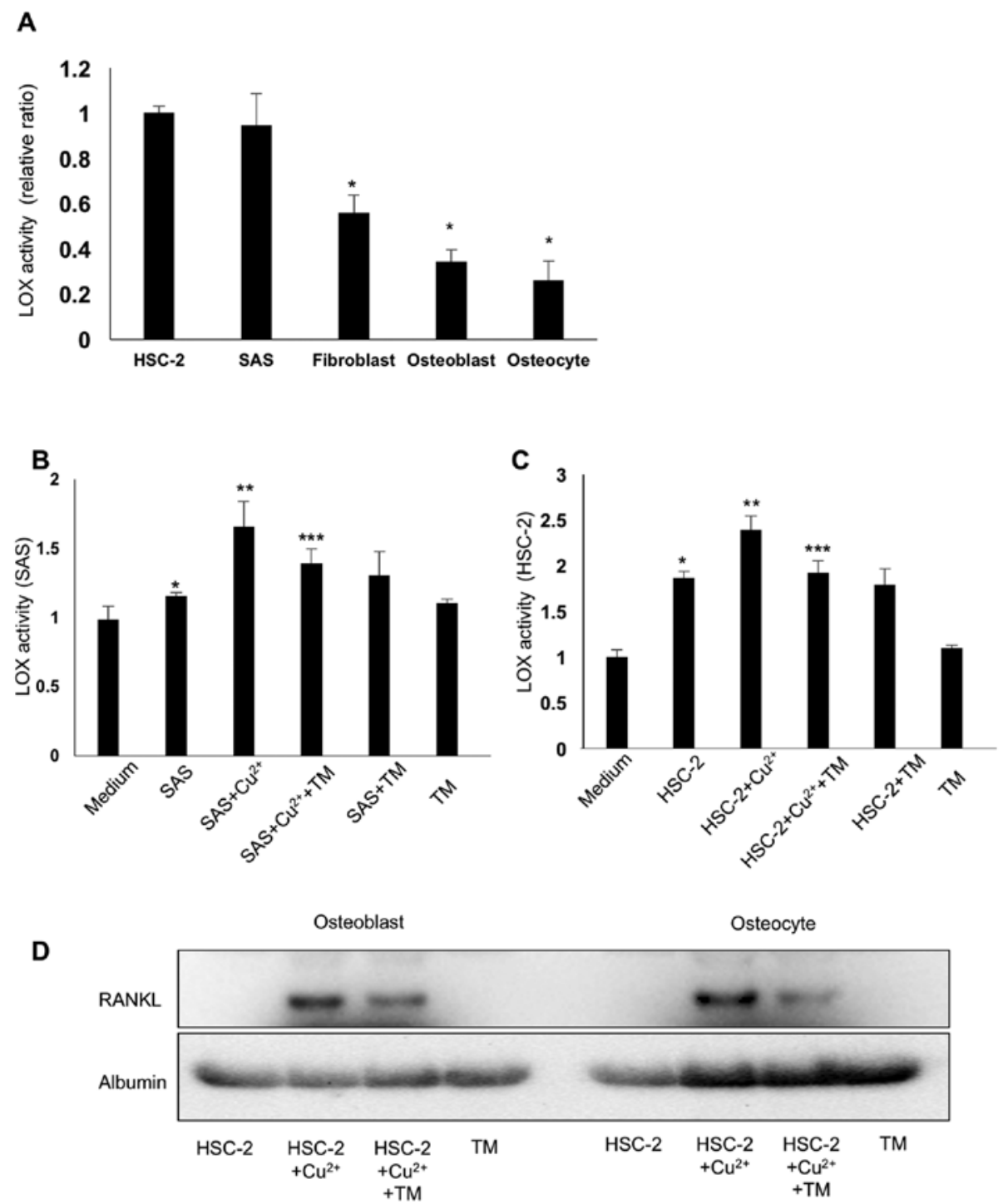

Figure 4. Effect of ammonium tetrathiomolybdate (TM) on LOX activity in cancer cells and the expression of RANKL in cells of the bone microenvironment. (A) LOX activation in conditioned media of HNSCC cells and bone microenvironmental cells. LOX activation is shown as the relative ratio. HSC-2 cells were used as a control (1.0). Data are the means $\pm \mathrm{SD} ;{ }^{*} \mathrm{P}<0.05$ vs. HSC-2 cells. (B) LOX activation in (B) SAS or (C) HSC-2 cell conditioned media with increased concentrations of copper ion $(10 \mu \mathrm{M})$ with or without $5 \mu \mathrm{M} \mathrm{TM}$. LOX activation is shown as the relative ratio. Medium was used as the control (1.0). Data are the means $\pm \mathrm{SD} ;{ }^{*} \mathrm{P}<0.05$ vs. medium, ${ }^{* *} \mathrm{P}<0.05$ vs. cancer $\mathrm{CM},{ }^{* * *} \mathrm{P}<0.05$ vs. $\mathrm{Cu}^{2+}$-added cancer $\mathrm{CM}$ (D) Osteoblasts and osteocytes were treated with the previously prepared HSC-2 conditioned medium for $24 \mathrm{~h}$. RANKL expression in the osteoblasts and osteocytes was evaluated by western blot analysis.

The copper concentration in the cell conditioned media and RANKL expression in bone marrow cells were decreased by TM treatment in a dose-dependent manner (Fig. 3C and D). By contrast, TM did not affect RANKL-induced osteoclast differentiation on CD11b-positive bone marrow cells (Fig. 3E). These results indicate that copper chelating by TM suppresses osteoclast formation via indirect osteoclast differentiation, such as that represented by RANKL expression in osteoblasts.

TM suppresses cancer cell-derived LOX activation via copper chelating. The enzyme activity of LOX was activated by copper binding to pro-LOX. Fig. 4A illustrates soluble LOX activation increased by copper ion $(10 \mu \mathrm{M})$ from the conditioned media of the cancer cells and bone microenvironment cells. Both the SAS and HSC-2 cells released a large amount of activated LOX compared to the bone microenvironment cells, i.e., the fibroblasts, osteoblasts and osteocytes. As expected,
TM suppressed LOX activation in the conditioned medium of HNSCC cells via copper chelating (Fig. 4B and C).

TM suppresses RANKL expression in osteoblasts and osteocytes in vitro. To examine the effects of copper-induced LOX activation on RANKL expression in bone marrow cells, we treated osteoblasts and osteocytes with HSC-2 conditioned medium and cultured the cells in increased copper ion concentrations with or without TM for $24 \mathrm{~h}$. The HSC-2 conditioned medium and the presence of increased copper ions promoted RANKL expression in the osteoblasts and osteocytes. TM treatment decreased this effect via copper chelating (Fig. 4D).

TM decreases the copper levels and cancer-induced LOX activity in vivo. To build on these findings, we evaluated the effects of TM on bone destruction and resorption induced by the injection of HSC-2 cells into mouse tibiae. The results 
A
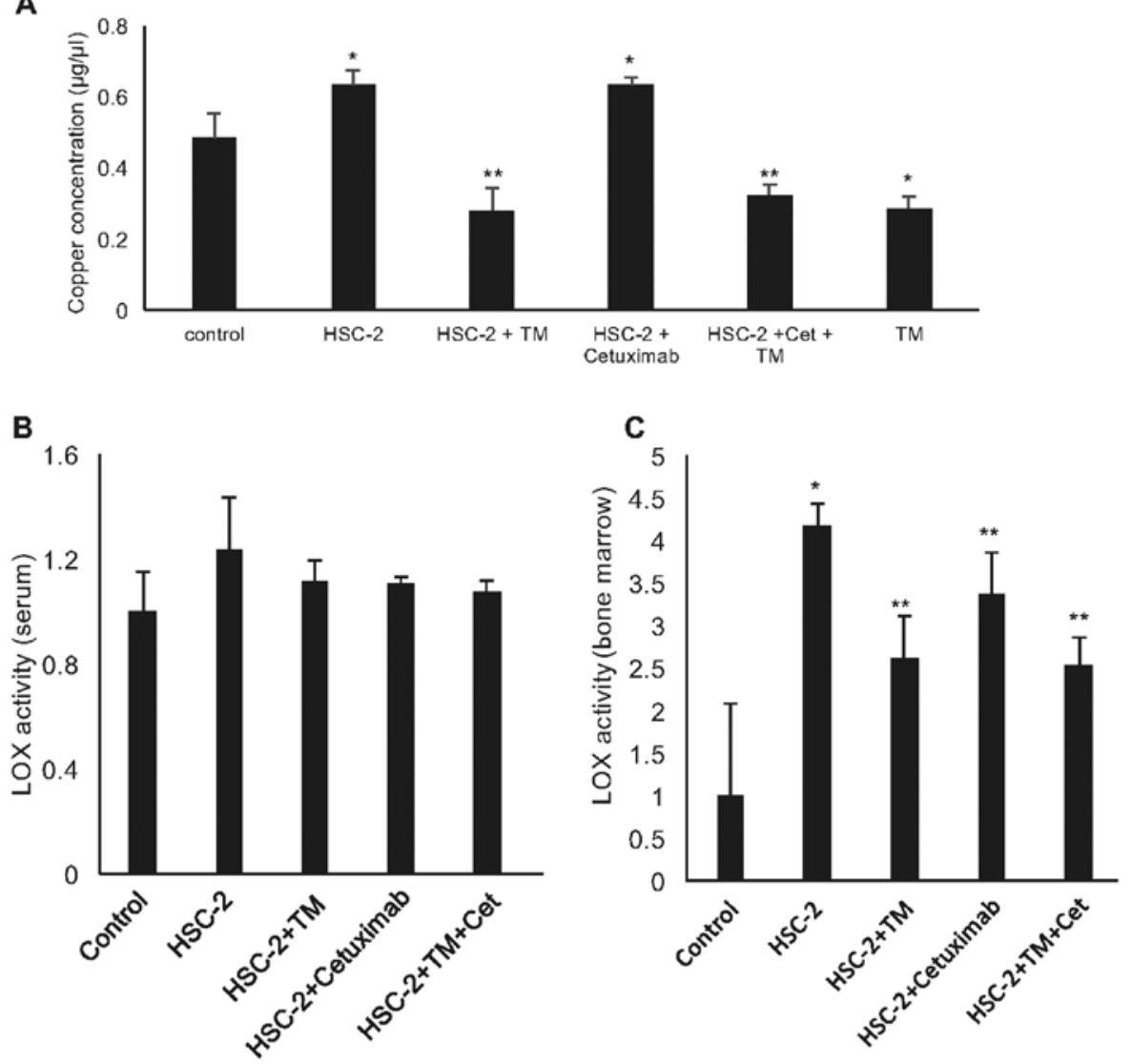

D

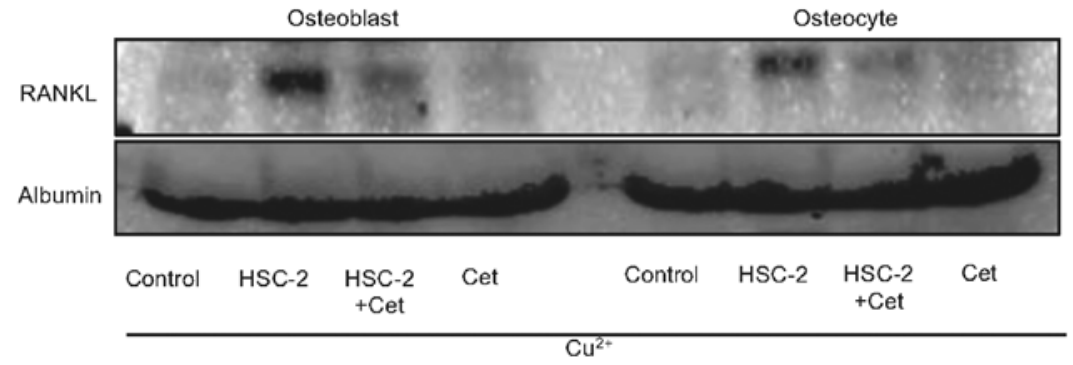

Figure 5. Effect of ammonium tetrathiomolybdate (TM) on the copper levels and LOX activity in free blood serum from mouse models of HSC-2-induced cancer bone invasion. At 7 days after tumor inoculation into he tibial bone marrow, mice were orally administered TM ( $1 \mathrm{mg} / \mathrm{body})$ or DDW 5 times a week for 5 weeks. Mice were intraperitoneally administered cetuximab $(1 \mathrm{mg} / \mathrm{kg}$ ) or PBS twice a week for 5 weeks (n=5/group). Copper levels in free blood and LOX activation in whole blood serum and bone marrow serum were measured in mice injected with the HSC-2 cells into their tibiae following treatment with TM or cetuximab alone or in combination. (A) Copper levels in free blood serum of the mouse model of bone invasion. Data are the means $\pm \mathrm{SD}$; ${ }^{*} \mathrm{P}<0.05 \mathrm{vs}$. controls, ${ }^{* *} \mathrm{P}<0.05$ vs. HSC-2 tumor cell-injected mice. (B) Analysis of LOX activity in whole blood serum. (C) Analysis of LOX activity analysis in bone marrow serum. LOX activation is shown as the relative ratio. Data are the means $\pm \mathrm{SD} ;{ }^{*} \mathrm{P}<0.05$ vs. control, ${ }^{* * *} \mathrm{P}<0.05$ vs. HSC-2 tumor cell-injected mice. (D) Osteoblasts and osteocytes were treated with the HSC-2 culture medium with increased concentrations of copper ion in the presence or absence of cetuximab for $24 \mathrm{~h}$. RANKL expression in osteoblasts and osteocytes was evaluated by western blot analysis.

demonstrated that the serum copper levels were increased in the mice injected with the HSC-2 into the tibiae, and this was suppressed by treatment with TM (Fig. 5A).

As expected, HSC-2 cell inoculation into the tibiae increased LOX activity in bone marrow, and treatment with TM and cetuximab significantly decreased this activity (Fig. 5C). However, TM did not affect the whole serum LOX activity (Fig. 5B). These data thus demonstrated that TM and cetuximab suppressed the local LOX activation in bone by chelating copper ion.

Cetuximab reduced LOX activation in bone marrow in vivo (Fig. 5C). We also evaluated the effects cetuximab treatment on RANKL expression in osteoblasts and osteocytes in vitro. Surprisingly, conditioned medium from the HSC-2 cells treated with cetuximab markedly decreased RANKL expression in osteoblasts and osteocytes (Fig. 5D). Cetuximab decreased the number of HNSCC cells in vitro and thereby cetuximab may suppress the amount of LOX in media.

TM enhances the anticancer effects of cetuximab and prevents bone resorption in vivo. We examined the in vivo effects of TM on osteolytic bone destruction induced by oral squamous carcinoma by conducting soft X-ray and micro-CT examinations. As shown in Fig. 6A and B, the osteolytic lesions were clearly visible in the tibiae of mice with bone invasion induced by HSC-2 cells treated with the vehicle only. Surprisingly, few destructive lesions were detected in the tibiae of mice treated with TM. The total area of radiographic osteolytic 
A

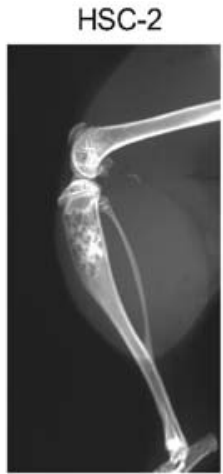

B

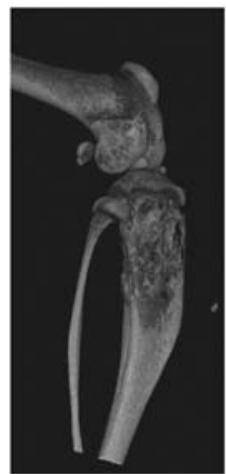

TM
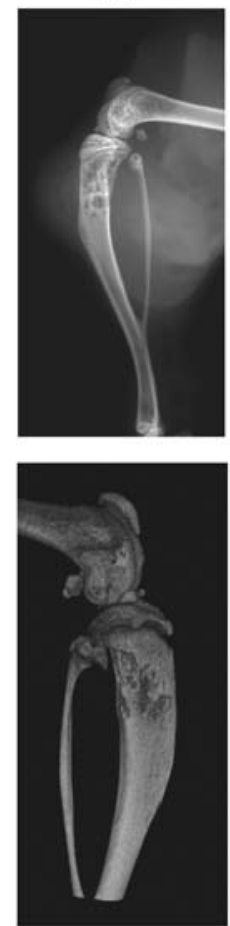

Cetuximab
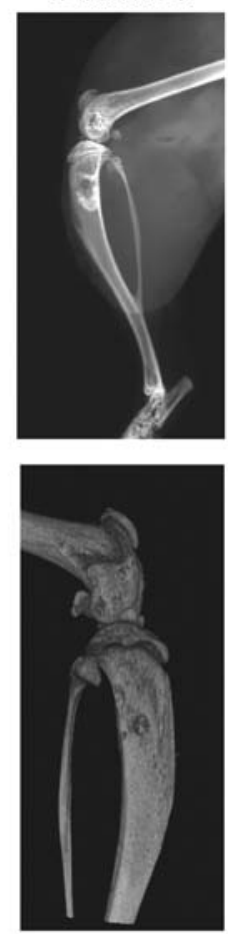
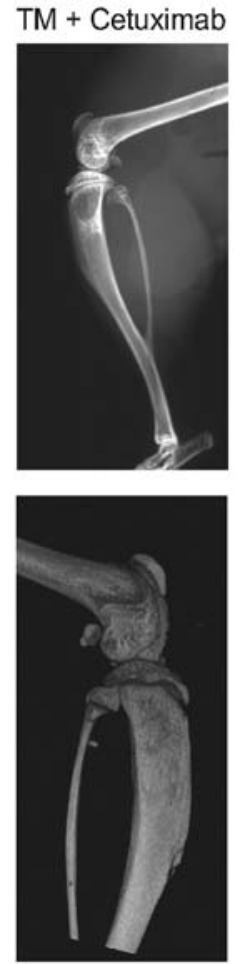

C

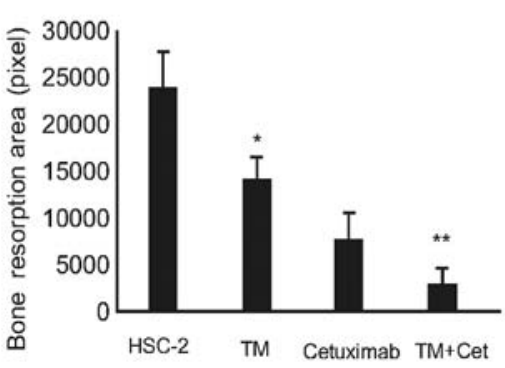

Figure 6. Effects of ammonium tetrathiomolybdate (TM) on mouse models of cancer-associated osteolysis. (A and B) Representative radiographs and micro-CT of tibia from mice with bone invasion following the injection of HSC-2 cells and treated with TM or cetuximab alone, or their combination ( $\mathrm{n}=5$ / group). (C) Osteolytic lesion area in tibia from mouse. The osteolytic area. Data are the means $\pm \mathrm{SD} ;{ }^{*} \mathrm{P}<0.05$ vs. HSC-2-injected mice; ${ }^{* *} \mathrm{P}<0.05$ vs. mice treated with cetuximab or TM alone.

lesions from all tibiae was significantly suppressed by TM treatment compared to the controls $(\mathrm{P}<0.05)$. Cetuximab is a standard of care agent for the treatment of human head and neck cancers (16), and it is well known to suppress HSC-2 cell growth. Surprisingly, TM enhanced the antitumor effects of cetuximab in bone (Figs. 6C and 7A). Treatment with both TM and cetuximab alone decreased cancer cell proliferation in bone marrow. Furthermore, combination treatment with TM and cetuximab intensively suppressed tumor cell proliferation in bone marrow compared to treatment with each agent alone.

In addition, the numbers of RANKL-positive cells and TRAP-positive osteoclasts were significantly decreased in the tibiae of the mice treated with both agents compared with the mice treated with single treatment (TM or cetuximab only) (P<0.05; Fig. 7B-D). To build on these findings, we examined the activity of cetuximab in this model. Cetuximab suppressed EGFR levels and phosphorylated EGFR expression in these bone marrow tumors. In addition, combined treatment with cetuximab and TM enhanced the suppressive effects on the epression of EGFR, phosphorylated EGFR, CD31 and Ki67 compared with single treatment (Fig. 8A).
These results suggest the following: i) TM significantly suppresses oral squamous cell carcinoma via the suppression of osteoclastogenesis and angiogenesis in osteolytic bone destruction that is associated with the invasion of oral squamous cell carcinoma; and ii) TM may enhance the effectiveness of cetuximab.

\section{Discussion}

Copper chelators have been previously reported to inhibit cancer cell growth in vitro and in vivo $(7,16,17)$. LOXs are copper-dependent enzymes. Copper ion binding to pro-LOX is necessary for LOX activation $(9,18)$. The most well-studied roles of LOX enzymes are in the remodeling of the ECM and angiogenesis. It has been reported that cancer-derived LOX induces bone destruction in HNSCC and other malignancies (19-21); however, the role of copper ion involvement in bone destruction induced by HNSCC is not yet well understood. To the best of our knowledge, the present findings are the first to demonstrate that the chelation of copper ions by TM, inhibited LOX activation from HSC-2 head and neck 
A

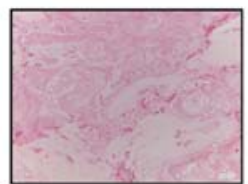

HSC-2

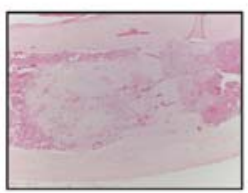

Cetuximab

C

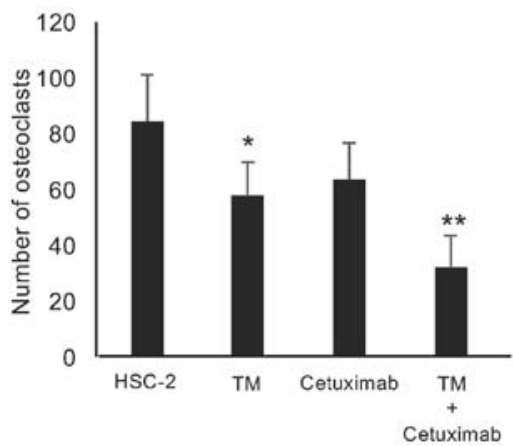

TRAP

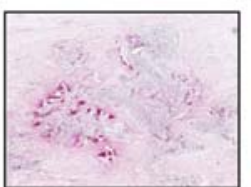

HSC-2

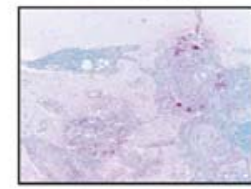

Cetuximab

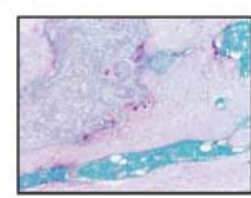

TM

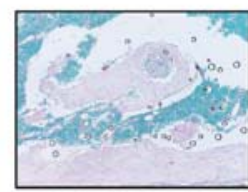

$\mathrm{TM}+$ Cetuximab

D

RANKL

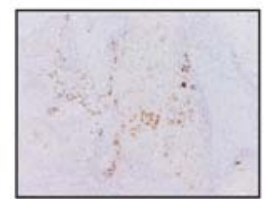

HSC-2

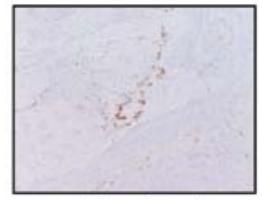

Cetuximab

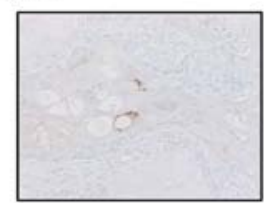

TM

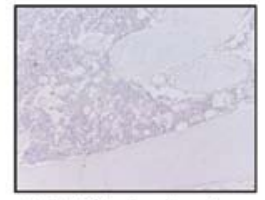

Figure 7. Histological and histomorphometric analysis of the bone marrow in the mouse model of bone invasion. The sections of previous-experiment mice tibial bone marrow were stained with (A) hematoxylin and eosin (H\&E), (B and C) TRAP and (D) RANKL (n=5/group). The numbers of osteoclasts in bone marrow are presented as thee means $\pm \mathrm{SD} ;{ }^{*} \mathrm{P}<0.05$ vs. control, ${ }^{* *} \mathrm{P}<0.05$ vs. treatment with ammonium tetrathiomolybdate (TM) or cetuximab alone.

A

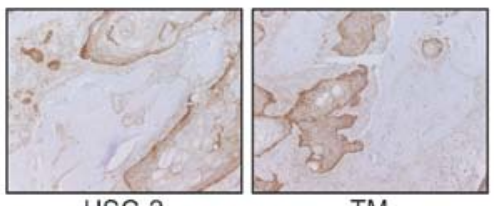

HSC-2

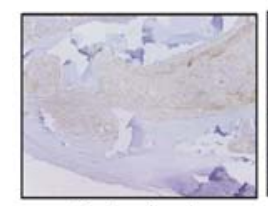

Cetuximab
EGFR

TM

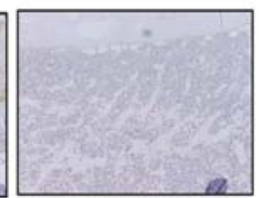

TM +Cetuximab
B

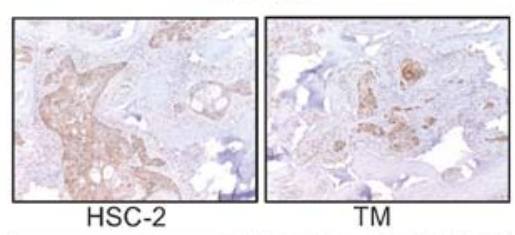

HSC-2

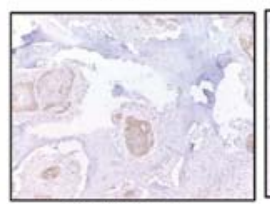

Cetuximab

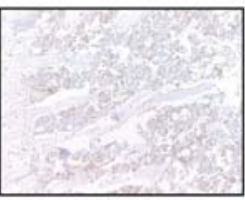

TM +Cetuximab

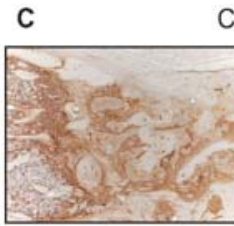

HSC-2

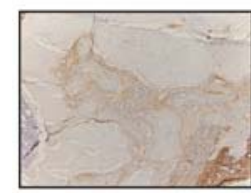

Cetuximab
CD31

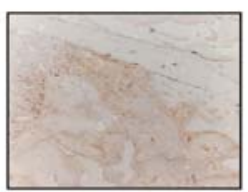

TM

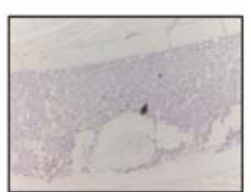

TM + Cetuximab

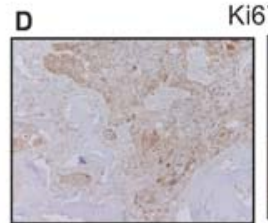

HSC-2

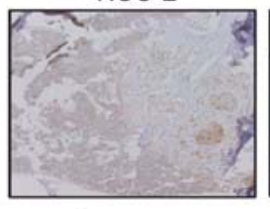

Cetuximab
Ki67

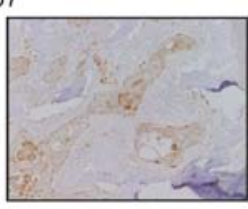

TM

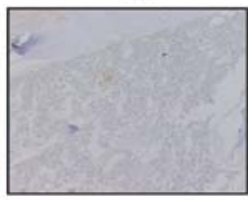

TM +Cetuximab

Figure 8. Immunohistological analysis of the bone marrow in the mouse model of bone invasion. Representative sections of mice tibial bone marrow stained with (A) EGFR, (B) p-EGFR, (C) CD31 and (D) Ki67 (n=5/group). 
cancer cell models. In an earlier study, LOX was shown to increase RANKL expression in osteoblasts and consequently promote osteoclastogenesis (11). In this study, we observed that copper chelation by TM inhibited RANKL expression in osteoblasts and osteocytes via LOX suppression, resulting in an inhibition of the bone destruction associated with HNSCC invasion. These results indicate that copper ions are a critical mediator of osteolytic bone destruction in the bone tumor microenvironment.

Our experiments revealed that the oral squamous cell carcinoma HSC-2 and SAS cells were potently and effectively inhibited by TM at the level of proliferation. TM inhibited HSC-2 and SAS cell growth at an $\mathrm{IC}_{50}$ of $1-5 \mu \mathrm{M}$, whereas TM did not inhibit the growth of the fibroblasts, osteocytes, osteoblasts or $\mathrm{T}$ cells at the $\mathrm{IC}_{50}$ of $5 \mu \mathrm{M}$ (Fig. 1D-G), which is consistent with previous findings (22). The discrepancy in $\mathrm{IC}_{50}$ results may be due to differences in the cellular systems, and cancer cells may be more sensitive to copper metabolism than normal cells. We also observed that the administration of TM exhibited efficacy in the HSC-2 xenograft model, where TM inhibited tumor growth in vivo. Furthermore, TM demonstrated a positive trend by enhancing the antitumor effects of cetuximab, which is an EGFR receptor inhibitor used to treat head and neck cancer patients (cetuximab single treatment vs. combined treatment, $\mathrm{P}=0.057$ ). However, there no statistically significant differences were observed between single treatment and combined treatment with TM and cetuximab as regards tumor volume in this soft tissue tumor model. These results suggest that the antitumor effects of TM require further evaluation in soft tissue HNSCC tumor models in vivo.

In our investigation of the molecular mechanisms of action of TM in osteoclastogenesis in total bone marrow cells and CD11b-positive bone marrow cells, the data indicated that TM inhibited osteoclastogenesis from total bone marrow cells induced by vitamin $\mathrm{D}_{3}$ (Fig. 3A and B). However, TM did not affect RANKL-induced osteoclast differentiation from CD11b-positive cells (Fig. 3E). These data indicated that TM did not have a direct effect on osteoclast precursor cells, but did have an effect on other cells of the bone microenvironment.

TM reduced LOX activation in the HSC-2 and SAS cells following copper ion treatment. It has been previously reported that LOX induces RANKL expression in osteoblasts (11). Consistently, the present findings demonstrated that the suppression of LOX downregulated RANKL expression in osteoblasts and osteocytes, resulting in the suppression of the differentiation of osteoclasts. The HSC-2 and SAS head and neck cancer cells released significant amounts of LOX (Fig. 4A). Copper ions increased LOX activation and TM suppressed it. These data indicated that TM may have a potent antitumor effect in bone-invasive HNSCC cells by not only suppressing tumor growth, but also by suppressing bone resorption by osteoclasts. To test this hypothesis, we created an HNSCC bone destruction mouse model, and treated the mice with TM. As expected, the serum copper levels in the mice injected with HSC-2 cells in their tibiae that were treated with $\mathrm{TM}$ were decreased compared to those of the untreated mice. The intratibial LOX activation in these mice was decreased similar to the serum copper levels. By contrast, there were no differences in LOX activation in blood serum. These data indicated that pro-LOX from cancer cells and copper ions are necessary for LOX activation and bone destruction.

Studies have reported that copper and LOX promote EGFR activation $(22,23)$. In the present study, TM inhibited EGFR and phosphorylated EGFR expression in HSC-2 tumors in vivo. This mechanism may result in reduced Ki67 expression and consequent tumor growth suppression. To test the effects of TM in vivo, we treated the mice in the bone-destructive HNSCC model with TM, cetuximab or both, and the results indicated that single treatment with TM and single treatment with cetuximab reduced tumor growth in the bone (Fig. 6), and the combination treatment significantly decreased tumor growth and bone resorption compared to single treatment with either agent alone. This additive effect was due to the suppression of osteoclast formation, angiogenic potential and EGFR activity (Figs. 7B-D, and 8A-C).

In conclusion, copper may be a target for the treatment of bone osteolysis induced by HNSCC, and our findings strongly suggest that the single use of TM or combination treatment with TM and approved agents, such as cetuximab requires further evaluation as a potential novel therapy for the treatment of advanced bone invasive HNSCC.

\section{Acknowledgements}

This study was supported by a Grant-in-Aid for Scientific Research (B) (JSPS KAKENHI grant no. 17H04405) to A. Sasaki and a Grant-in-Aid for Research Activity start-up (JSPS KAKENHI grant no. 16H06992) to T. Okui from the Ministry of Education, Culture, Sports, Science, and Technology of Japan.

\section{Competing interests}

The authors declare that they have no competing interests.

\section{References}

1. Brown JS, Lowe D, Kalavrezos N, D'Souza J, Magennis P and Woolgar J: Patterns of invasion and routes of tumor entry into the mandible by oral squamous cell carcinoma. Head Neck 24: 370-383, 2002.

2. Shaw RJ, Brown JS, Woolgar JA, Lowe D, Rogers SN and Vaughan ED: The influence of the pattern of mandibular invasion on recurrence and survival in oral squamous cell carcinoma. Head Neck 26: 861-869, 2004.

3. Takada H, Ibaragi S, Eguchi T, Okui T, Obata K, Masui M, Morisawa A, Takabatake K, Kawai H, Yoshioka N, et al: Semaphorin 4D promotes bone invasion in head and neck squamous cell carcinoma. Int J Oncol 51: 625-632, 2017.

4. Spence JA, Suttle NF, Wenham G, El-Gallad T and Bremner I: A sequential study of the skeletal abnormalities which develop in rats given a small dietary supplement of ammonium tetrathiomolybdate. J Comp Pathol 90: 139-153, 1980.

5. Smith BJ, King JB, Lucas EA, Akhter MP, Arjmandi BH and Stoecker BJ: Skeletal unloading and dietary copper depletion are detrimental to bone quality of mature rats. J Nutr 132: 190-196, 2002.

6. Roberts EA and Schilsky ML; American Association for Study of Liver Diseases (AASLD): Diagnosis and treatment of Wilson disease: An update. Hepatology 47: 2089-2111, 2008.

7. Chan N, Willis A, Kornhauser N, Ward MM, Lee SB, Nackos E, Seo BR, Chuang E, Cigler T, Moore A, et al: Influencing the tumor microenvironment: A phase II study of copper depletion using tetrathiomolybdate in patients with breast cancer at high risk for recurrence and in preclinical models of lung metastases. Clin Cancer Res 23: 666-676, 2017. 
8. Hassouneh B, Islam M, Nagel T, Pan Q, Merajver SD and Teknos TN: Tetrathiomolybdate promotes tumor necrosis and prevents distant metastases by suppressing angiogenesis in head and neck cancer. Mol Cancer Ther 6: 1039-1045, 2007.

9. Bhuvanasundar R, John A, Sulochana KN, Coral K, Deepa PR and Umashankar V: A molecular model of human Lysyl Oxidase (LOX) with optimal copper orientation in the catalytic cavity for induced fit docking studies with potential modulators Bioinformation 10: 406-412, 2014

10. Rucker RB, Kosonen T, Clegg MS, Mitchell AE, Rucker BR, Uriu-Hare JY and Keen CL: Copper, lysyl oxidase, and extracellular matrix protein cross-linking. Am J Clin Nutr 67 (Suppl): S996-S1002, 1998.

11. Tsukasaki M, Hamada K, Okamoto K, Nagashima K, Terashima A, Komatsu N, Win SJ, Okamura T, Nitta T, Yasuda H, et al: LOX fails to substitute for RANKL in osteoclastogenesis. J Bone Miner Res 32: 434-439, 2017.

12. Cox TR, Rumney RMH, Schoof EM, Perryman L, Høye AM, Agrawal A, Bird D, Latif NA, Forrest H, Evans HR, et al: The hypoxic cancer secretome induces pre-metastatic bone lesions through lysyl oxidase. Nature 522: 106-110, 2015.

13. Reynaud C,Ferreras L, Di Mauro P, Kan C, Croset M, Bonnelye E, Pez F, Thomas C, Aimond G, Karnoub AE, et al: Lysyl oxidase is a strong determinant of tumor cell colonization in bone. Cancer Res 77: 268-278, 2017

14. Teramachi J, Nagata Y, Mohammad K, Inagaki Y, Ohata Y, Guise T, Michou L, Brown JP, Windle JJ, Kurihara N, et al: Measles virus nucleocapsid protein increases osteoblast differentiation in Paget's disease. J Clin Invest 126: 1012-1022, 2016.

15. Shah KM, Stern MM, Stern AR, Pathak JL, Bravenboer N and Bakker AD: Osteocyte isolation and culture methods. Bonekey Rep 5: 838, 2016.
16. Park SJ, Kim MJ, Kim YK, Kim SM, Park JY and Myoung H: Combined cetuximab and genistein treatment shows additive anti-cancer effect on oral squamous cell carcinoma. Cancer Lett 292: 54-63, 2010 .

17. Chisholm CL, Wang H, Wong AH, Vazquez-Ortiz G, Chen W, $\mathrm{Xu} \mathrm{X}$ and Deng CX: Ammonium tetrathiomolybdate treatment targets the copper transporter ATP7A and enhances sensitivity of breast cancer to cisplatin. Oncotarget 7: 84439-84452, 2016.

18. Krebs CJ and Krawetz SA: Lysyl oxidase copper-talon complex: A model. Biochim Biophys Acta 1202: 7-12, 1993.

19. Shih YH, Chang KW, Chen MY, Yu CC, Lin DJ, Hsia SM, Huang HL and Shieh TM: Lysyl oxidase and enhancement of cell proliferation and angiogenesis in oral squamous cell carcinoma. Head Neck 35: 250-256, 2013.

20. Cox TR, Gartland A and Erler JT: Lysyl oxidase, a targetable secreted molecule involved in cancer metastasis. Cancer Res 76: 188-192, 2016.

21. Gartland A, Erler JT and Cox TR: The role of lysyl oxidase, the extracellular matrix and the pre-metastatic niche in bone metastasis. J Bone Oncol 5: 100-103, 2016.

22. Kim KK, Han A, Yano N, Ribeiro JR, Lokich E, Singh RK and Moore RG: Tetrathiomolybdate mediates cisplatin-induced p38 signaling and EGFR degradation and enhances response to cisplatin therapy in gynecologic cancers. Sci Rep 5: 15911, 2015.

23. Tang H, Leung L, Saturno G, Viros A, Smith D, Di Leva G Morrison E, Niculescu-Duvaz D, Lopes F, Johnson L, et al: Lysyl oxidase drives tumour progression by trapping EGF receptors at the cell surface. Nat Commun 8: 14909, 2017. 